\title{
Antianginal and anti-ischaemic efficacy of tedisamil, a potassium channel blocker
}

K M Fox, J R Henderson, J C Kaski, A Sachse, L Kuester, S Wonnacott, on behalf of the Third Clinical European Studies in Angina and Revascularisation (CESAR 3) Investigators

\begin{abstract}
Objective-To determine the efficacy and safety of the potassium channel blocker tedisamil versus placebo in the treatment of patients with stable angina.

Design-Prospective, double blind, placebo controlled study. 203 patients first completed a seven day placebo run in. They were then randomised to receive $50 \mathrm{mg}, 100 \mathrm{mg}$ or $150 \mathrm{mg}$ tedisamil twice daily, or placebo. Treadmill exercise testing was carried out at baseline and after 14 days of double blind treatment.

Main outcome measures-Primary efficacy parameters were an increase in total exercise duration and a reduction of the sum of ST segment depression using six ECG leads at maximum workload at trough (12 hours after last medication). Secondary aims included increase in exercise time to onset of $0.1 \mathrm{mV} \mathrm{ST} \mathrm{segment} \mathrm{depression,} \mathrm{increase} \mathrm{in} \mathrm{exercise} \mathrm{time} \mathrm{to} \mathrm{onset} \mathrm{of} \mathrm{any} \mathrm{anginal}$ pain, and reduction in ST segment depression in any of the six specified leads at maximum workload. These were all at trough. The same parameters were also assessed at peak concentrations (two hours after administration). Overall attacks of angina and the use of short acting nitrates were assessed from patient diaries.

Results-Tedisamil led to a dose dependent prolongation of exercise duration (significant at all concentrations), an effect that was greater at peak than at trough. Treatment also led to a significant dose dependent reduction in the sum of ST segment depression at both trough and peak concentrations. Tedisamil also decreased (in a dose dependent way) the frequency of anginal attacks and the consumption of short acting nitrates, an improvement that became significant for all doses in the second treatment week. Adverse events with tedisamil were few. There was a pronounced rise in the incidence of diarrhoea with the $150 \mathrm{mg}$ twice daily regimen. Bradycardic effects and increases in QT interval were dose dependent, but were no more evident at exercise than at rest.

Conclusions-Tedisamil, at doses of 50-100 mg twice daily, was found to be an effective antianginal and anti-ischaemic agent. At doses above $100 \mathrm{mg}$ twice daily its main side effect, diarrhoea, becomes pronounced; therefore the 50-100 mg twice daily regimen appears to be appropriate.

(Heart 2000;83:167-171)
\end{abstract}

Keywords: stable angina; potassium channel blocker, tedisamil

Tedisamil is a bradycardic drug that is being investigated for use in the treatment of angina. It is a potassium channel blocker that inhibits both transient outward $\left(1_{\text {to }}\right)$ and delayed rectifier $\left(1_{k}\right)$ potassium currents. ${ }^{12}$ Chemically it is a heterocyclic dihydrochloride derivative of sparteine, and is unrelated to any known antianginal drug. It has a bioavailability of about $50 \%$ and is excreted unchanged by the kidneys, with an elimination half life of about 10 hours.

Tedisamil's bradycardic activity stems from a dose dependent QTc interval prolongation, particularly a prolongation of the repolarisation phase in pacemaker cells of the sinus node. Animal and human studies have shown no modification of cardiac contractility or relaxation, with clear anti-ischaemic effects in its therapeutic dose range in humans (50-200 mg twice daily).

The effects on lengthening QTc have also led to investigation of tedisamil as a class III antiarrhythmic agent, and further studies are in progress. The present paper, however, is concerned only with the drug's antianginal and anti-ischaemic effects. A phase I programme of oral and intravenous dosing has shown a dose dependent decrease in heart rate as well as QT prolongation, which remained after correction for heart rate. The bradycardic effect became obvious at a dose of $8 \mathrm{mg}$ intravenously and $>100 \mathrm{mg}$ by mouth. The bradycardic and QTc prolonging effects were similar under resting and exercise conditions.

In a preliminary dose ranging placebo controlled trial (with 115 patients analysed so far) the anti-ischaemic and antianginal effects of tedisamil have been investigated in patients with ischaemic heart disease and angina. The sum of ST segment depression (leads II, III, aVF, $V_{4}, V_{5}$, and $V_{6}$ ) was the primary efficacy variable, and was reduced dose dependently by tedisamil. This became significant compared to placebo at dosages of $100 \mathrm{mg}$ and $200 \mathrm{mg}$ twice daily. The lowest dosage of tedisamil used in this trial, $50 \mathrm{mg}$ twice daily, showed a reduction of the sum of ST segment depression compared to placebo; this difference was not significant. Adverse events were more common in patients taking tedisamil versus placebo. The 
most common event reported was diarrhoea, which was dose dependent. Serious adverse events were more common in the patients on placebo than in those taking tedisamil.

\section{Methods}

Our study's objective was to determine the antianginal and anti-ischaemic effects of $50 \mathrm{mg}, 100 \mathrm{mg}$, and $150 \mathrm{mg}$ tedisamil twice daily at peak and trough concentration after two weeks' treatment, compared with baseline and placebo. The primary efficacy parameters were an increase in time to angina and reduction of the sum of $S T$ segment depression over six leads at maximum workload for each patient. These assessments were made at trough, 12 hours after last drug intake. The secondary aims were: the primary aims, but calculated at peak concentration (two hours after dosing); the increase in time to onset of $0.1 \mathrm{mV}$ ST segment depression during exercise testing at peak and trough; and the reduction of maximum ST segment depression in any of the specified leads at each patient's maximum workload.

Reduction in frequency of anginal attacks and consumption of short acting nitrates was recorded in a patient diary. All adverse events were recorded by the investigator.

The study randomised 226 ambulatory patients with chronic, stable, effort induced angina pectoris at 35 centres throughout the UK. Two hundred and three patients completed the study, which was done in accordance with European Council of Good Clinical Practice guidelines, the Helsinki Convention, and with the approval of local ethics committees.

The inclusion criteria allowed patients of either sex older than 18 years and suffering from chronic stable angina pectoris to be entered into the study. Their myocardial ischaemia was clinically demonstrable at ECG, and they were required to have a positive reproducible exercise test during the placebo run in period.

CONCURRENT MEDICATION

Patients taking antianginal medication were not admitted to the study unless there was a wash out period of at least five half lives (for $\beta$ blockers seven half lives) of the respective drug. Forbidden antianginal drugs were: $\beta$ blockers (8-10 days), calcium channel blockers (normally 2-3 days, for amlodipine 10 days), and long acting organic nitrates (2-3 days). Digitalis (at least 10 days), dipyridamole (2-3 days), and amiodarone (at least six months' washout) or other antiarrhythmic agents (2-3 days) were also forbidden. A protocol amendment subsequently excluded the use of terfenadine, ketoconazole, amiodarone, class I antiarrhythmics, or any other drugs known to prolong the QT interval.

Short acting nitrates (glyceryl trinitrate) were permitted during the trial, while their time of use and dosage were documented in patient diaries. All other medication was documented and kept constant throughout the trial period. Duration of intake of all co-medication was precisely documented in the patients' case record forms. When an anginal attack involving treatment with a short acting nitrate occurred during the two hour period before exercise testing, the testing procedure was performed later, if in the responsible doctor's opinion there was no risk for the patient and two hours had passed after nitrate use.

\section{PROCEDURES}

All visits started so that the treadmill tests could be performed at 10:00 plus or minus one hour for efficacy assessments. This was about 12 hours after intake of the last (evening) dose of study medication. For each patient recordings were made at the same time of day.

The study began with a screening visit not more than two weeks before the beginning of the single blind run in period; the results of the screening visit needed to be known by the investigator before the patient entered the run in period. All antianginal and other prohibited medications were withdrawn and washed out before visit 1 (short acting nitrates were acceptable). The assessments at the screening visit included: demographic data, compliance with inclusion/exclusion criteria, medical history, physical examination, blood pressure, weight, haematology, blood chemistry, urinalysis, tedisamil plasma concentration (blank), 12 lead ECG and heart rate, concurrent medication and baseline complaints, assessment of alcohol, and tobacco and caffeine consumption. Written informed consent was obtained at this time.

After establishing the suitability of the patient, an appointment was made for day 1 (visit 1) when the patient was given placebo medication for the run in phase, a treadmill test (Bruce protocol), a 12 lead ECG, and was questioned about baseline complaints and concurrent medication. After the treadmill test at 10:00, patients were given their event diary so that they could record drug intake, angina attacks, use of nitrates, and any other signs or symptoms.

The run in phase lasted seven days; patients were instructed to take their study medication with food at 10:00 and 22:00 regularly. At the end of the seven days, they returned for visit 2 (day 8) when their blood pressure and 12 lead ECG were recorded, and their medication, adverse events, and diaries checked. After this a treadmill test was performed with the patients not having taken any medication. They could then proceed to the double blind period of the trial, provided that: the two treadmill tests under placebo did not differ by more that $20 \%$ with regard to the exercise duration; an ST segment depression of at least $0.1 \mathrm{mV}$ occurred in any lead under both tests while taking placebo; the two treadmill tests were terminated because of angina; and patients showed adequate compliance in taking the study medication.

Randomisation (at visit 2) allocated patients to one of four treatment groups: tedisamil $50 \mathrm{mg}$ twice daily; tedisamil $100 \mathrm{mg}$ twice daily; tedisamil $150 \mathrm{mg}$ twice daily; placebo twice daily. 
After a baseline treadmill test at 10:00 at visit 2 , patients took their first dose of double blind medication and remained at the investigational site for two hours after drug intake. Sitting blood pressure, as well as ECG, was measured to exclude an excessive drug response after the first dose. If any excessive drug response (such as bradycardia or excessive QT prolongation) occurred, it was the investigator's decision whether or not to continue the patient in the study. Out of 403 patients screened 226 were randomised to one of the four treatment groups. Visit 3 occurred on day 15, which was the eighth day of randomised treatment. Assessments were made as at visit 2, followed by a treadmill test at 10:00. Visit 4 occurred on day 22 after two weeks of randomised treatment. Assessments were made as with visits 2 and 3, and a treadmill test performed at 10:00. Directly after this, the patients took their last dose of study medication and two hours later a second treadmill test was performed to assess the effects of the drug at peak plasma concentration. The other four tests in the study were all at trough concentration. Visit 5 occurred on days 23-27 (poststudy assessment). The standard assessments (visits 2, 3, and 4) were performed without a treadmill test.

HOLTER ECG MONITORING

Twenty four hour ECG monitoring was carried out for all patients at day 1 , day 8 , and visit 4 (day 22) in order to minimise the risk of including patients with pre-existing arrhythmia, and to allow patients to be withdrawn should serious arrhythmia occur. In fact, no arrhythmia was seen.

STATISTICS

The sample size was calculated to satisfy the following assumptions (as stated in the protocol): a clinically relevant difference of $0.15 \mathrm{mV}$ for the sum of ST segment depression with an SD of $0.21 \mathrm{mV}$; a type 1 error probability $(\alpha$ level) of $2.5 \%$ and a power of $80 \%$.

Given these assumptions, the required sample size was calculated to be 204-that is, 51 patients per treatment group.

Three main patient cohorts were identified for the analyses:

(1) The safety patient cohort $(n=226)$, comprising all patients who were randomised into the double blind treatment period, who had taken double blind treatment at least once, and for whom at least some

Table 1 Demographic information: safety patient group $(n=226)$

\begin{tabular}{|c|c|c|c|c|c|}
\hline & \multirow[b]{2}{*}{$\begin{array}{l}\text { Placebo } \\
(n=57)\end{array}$} & \multicolumn{3}{|c|}{ Tedisamil twice daily } & \multirow[b]{2}{*}{$\begin{array}{l}\text { Total } \\
(n=226)\end{array}$} \\
\hline & & $\begin{array}{l}50 \mathrm{mg} \\
(n=55)\end{array}$ & $\begin{array}{l}100 \mathrm{mg} \\
(n=60)\end{array}$ & $\begin{array}{l}150 \mathrm{mg} \\
(n=54)\end{array}$ & \\
\hline Age (years) & $59.7(6.5)$ & $60.5(7.4)$ & $61.7(6.8)$ & $60.7(7.3)$ & $60.7(7.0)$ \\
\hline \multicolumn{6}{|l|}{$\operatorname{Sex}(\mathrm{n}(\%))$} \\
\hline Male & $56 \quad(98.2)$ & $50 \quad(90.9)$ & $54 \quad(90.0)$ & $(92.6)$ & $(92.9)$ \\
\hline Female & $(1.8)$ & $(9.1)$ & $(10.0)$ & $(7.4)$ & $(7.1)$ \\
\hline \multicolumn{6}{|l|}{ Weight $(\mathrm{kg})$} \\
\hline Male & $80.3(12.4)$ & $81.3(9.2)$ & $83.0(11.0)$ & $79.8(11.7)$ & $81.1(11.2)$ \\
\hline Female & 79.0 & $61.6(14.8)$ & $75.3(10.8)$ & $69.8(14.2)$ & $69.9(13.3)$ \\
\hline \multicolumn{6}{|l|}{ Height $(\mathrm{cm})$} \\
\hline Male & 173 & 171 & 173 & (15) & (10) \\
\hline Female & 170 & 159 & 161 & 160 & 161 \\
\hline
\end{tabular}

Values are mean (SD) except where stated. information concerning safety parameters had been recorded.

(2) Intent to treat cohort $(n=211)$, being a subset of the safety patient cohort, consisted of all randomised patients with an evaluable exercise test at trough on day 15 or day 22.

(3) Per protocol patient cohort $(n=111)$, comprising a subset of the intent to treat cohort, included patients without major protocol violations. This cohort was used for efficacy analysis only.

\section{Results}

The demographics of the study population are shown in table 1 . They are similar in all treatment cohorts. The majority of patients were male.

Sixty three per cent of the patients were former smokers, $72.1 \%$ reported moderate alcohol intake, and $73.9 \%$ were moderate coffee or tea drinkers. Coronary angiography had been performed in $45.6 \%$ of the patients; $35.05 \%$ suffered from one vessel disease, $37.1 \%$ from two vessel disease, and $27.8 \%$ from three or more vessel disease. A history of myocardial infarction was reported by $29.6 \%$ of the patients but only $18.7 \%$ had undergone cardiovascular intervention or surgery.

PRIMARY EFFICACY PARAMETERS

Sum of ST segment depression

When assessed in the intent to treat cohort, the reductions in the sum of ST segment depression were $-0.05 \mathrm{mV},-0.12 \mathrm{mV},-0.13 \mathrm{mV}$, and $-0.13 \mathrm{mV}$ for placebo, $50 \mathrm{mg}, 100 \mathrm{mg}$, and $150 \mathrm{mg}$ tedisamil twice daily, respectively. These improvements were not significant, although they came close to the (predefined) clinically relevant effect of $0.15 \mathrm{mV}$ reduction in all three active treatment groups. In the preprotocol cohort, however, the reductions were $-0.01 \mathrm{mV}, \quad-0.18 \mathrm{mV}, \quad-0.18 \mathrm{mV}, \quad$ and $-0.20 \mathrm{mV}$ for placebo, $50 \mathrm{mg}, 100 \mathrm{mg}$, and $150 \mathrm{mg}$ tedisamil twice daily, respectively, reaching significance and exceeding the threshold of a clinically relevant effect with all tedisamil doses.

We have chosen, however, to illustrate the findings in the per protocol cohorts, for these included those patients showing $0.1 \mathrm{mV} \mathrm{ST}$ depression at baseline - an important disease defining inclusion criterion. This seems a more appropriate cohort to use (fig 1). This figure also shows the effect of tedisamil at peak (two hours after dosing). This is a secondary aim of the study and shows significant improvement with all three dose regimens versus placebo.

\section{Increase in time to moderate anginal chest pain} (duration of exercise)

In the intent to treat cohort the increase in time was $0.62 \mathrm{~min}, 0.71 \mathrm{~min}, 0.92 \mathrm{~min}$, and 1.04 min for patients receiving placebo, $50 \mathrm{mg}$, $100 \mathrm{mg}$, and $150 \mathrm{mg}$ tedisamil twice daily, respectively. Significant linear dose dependency was observed using all treatments, and pairwise comparison versus placebo was significant for the highest dose. The per protocol 
$\square 2$ hours post (peak) $\square 12$ hours post (trough)

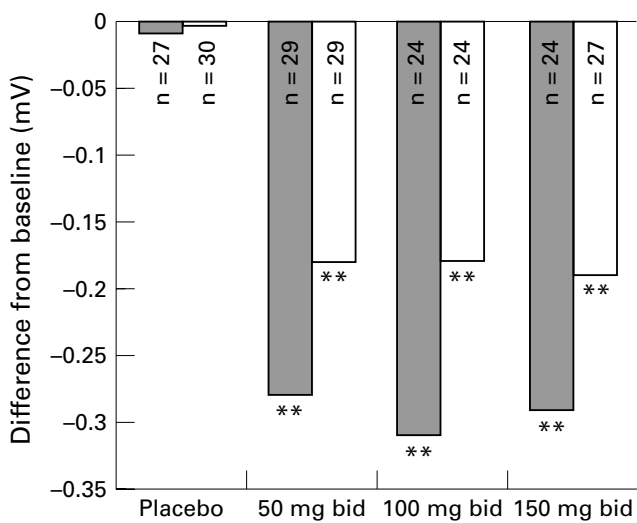

Figure 1 Sum of ST segment depression at highest comparable workload at peak and trough plasma concentrations (per protocol group). ${ }^{\star \star} p<0.01$

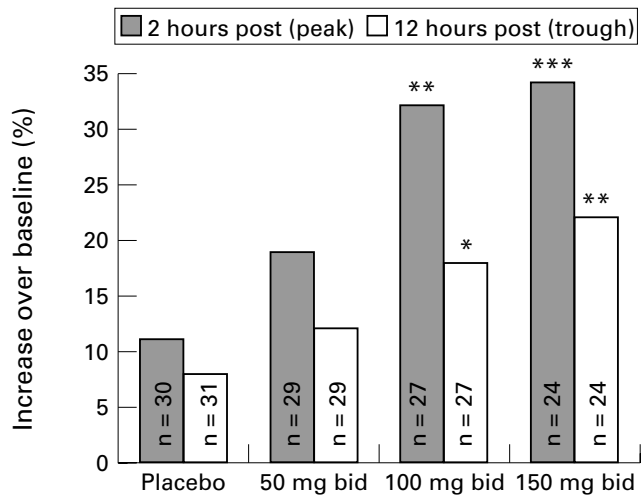

Figure 2 Duration of exercise at peak and trough plasma concentrations (per protocol group). Significant unadjusted $p$ values: ${ }^{\star} p<0.05 ;{ }^{\star *} p<0.01 ;{ }^{\star *} p<0.001$.

cohort showed significant differences that were even more pronounced (fig 2).

PATIENT DIARIES

For the evaluation of the diaries, only those patients in the safety patient group with positive findings in the run in period-that is, with one or more anginal attacks and nitrate consumption-were considered.

The reduction in the frequency of anginal attacks was greater in the tedisamil groups than in the placebo group. During the first week of treatment, the reduction in anginal attacks was significant only in the $150 \mathrm{mg}$ twice daily group versus placebo, with a trend in the $100 \mathrm{mg}$ twice daily group. During the second week, however, the reduction was significant for the $50 \mathrm{mg}$ and $150 \mathrm{mg}$ twice daily groups versus placebo, with a trend in the $100 \mathrm{mg}$ twice daily group.

A similar effect was seen in decreasing nitrate consumption. The median weekly intake of nitrates was reduced during the course of double blind treatment from 5 to 2.3 units with tedisamil $50 \mathrm{mg}$ twice daily, from 5 to 1 unit with $100 \mathrm{mg}$ twice daily, and from 5 to 1 unit with $150 \mathrm{mg}$ twice daily. These results reached significance for all three tedisamil groups (when compared with placebo) in the second week.
SAFETY PARAMETERS.

Adverse events associated with placebo numbered $22 / 57(38.6 \%)$, with $50 \mathrm{mg}$ tedisamil twice daily $21 / 55(38.2 \%)$, with $100 \mathrm{mg}$ twice daily $33 / 60(55 \%)$, and with $150 \mathrm{mg}$ twice daily $39 / 54(72.7 \%)$. Diarrhoea was the most common adverse event associated with tedisamil, and was dose dependent. In the safety patient group $(\mathrm{n}=226)$ the incidence of diarrhoea was $0 \%, 3.6 \%, 13.3 \%$, and $44.4 \%$ in patients taking placebo, $50 \mathrm{mg}, 100 \mathrm{mg}$, and $150 \mathrm{mg}$ tedisamil twice daily, respectively.

No death was reported during the study. Serious adverse events were seen in 14 patients. Six of these were before randomisation, and eight after (four patients taking placebo and four tedisamil). The serious adverse events after randomisation included collapse (placebo), chest pain (placebo), three cases of ventricular tachycardia (placebo, $50 \mathrm{mg}$, and $100 \mathrm{mg}$ tedisamil twice daily,), two cases of unstable angina (placebo and $100 \mathrm{mg}$ tedisamil twice daily), and crescendo angina (100 mg tedisamil twice daily).

Withdrawals because of adverse events occurred in 18 patients after randomisation, including seven patients with serious adverse events. The withdrawal rates were $4 / 57(7 \%)$, $1 / 55(1.8 \%), 4 / 60(6.7 \%)$, and $9 / 54(16.7 \%)$ for the placebo, $50 \mathrm{mg}, 100 \mathrm{mg}$, and $150 \mathrm{mg}$ tedisamil twice daily groups, respectively; in this last group 5/54 (9.3\%) patients withdrew because of diarrhoea.

\section{Bradycardic effects}

At two hours postdose, bradycardic effects were observed with all three dose regimens of tedisamil. On day 8, the heart rate (beats per minute) at two hours postdose for patients receiving placebo was $3.0 \mathrm{bpm}$ less than baseline, and $6.5 \mathrm{bpm}, 9.3 \mathrm{bpm}$, and $10.6 \mathrm{bpm}$ less than baseline for patients received $50 \mathrm{mg}$, $100 \mathrm{mg}$, and $150 \mathrm{mg}$ tedisamil twice daily, respectively. Under steady state conditions at trough (days 15 and 22) the heart rate lowering effect was minimal in all the tedisamil treatment groups when compared to placebo.

\section{Effects on cardiac repolarisation}

Tedisamil prolonged the QT interval by 3, 11, and $18 \mathrm{~ms}$ for the three dose regimens of tedisamil; with placebo the QT was shortened by $-2 \mathrm{~ms}$. None of these effects were significant. After correction for heart rate using Bazett's formula, a QTc prolongation of $2 \mathrm{~ms}, 0 \mathrm{~ms}$, $11 \mathrm{~ms}$, and $17 \mathrm{~ms}$ was calculated for placebo, $50 \mathrm{mg}, 100 \mathrm{mg}$, and $150 \mathrm{mg}$ tedisamil twice daily, respectively. The mean maximum QTC effects at any time under treatment were $14 \mathrm{~ms}, 15 \mathrm{~ms}, 35 \mathrm{~ms}$, and $33 \mathrm{~ms}$, for the placebo and three dose regimens of tedisamil, respectively, these effects being significant for the $100 \mathrm{mg}$ and $150 \mathrm{mg}$ tedisamil twice daily groups. A QTc value exceeding $0.48 \mathrm{~ms}$ under treatment was observed in 21 patients: two with placebo, one with $50 \mathrm{mg}$, eight with $100 \mathrm{mg}$, and 10 with $150 \mathrm{mg}$ tedisamil twice daily. No changes in other ECG parameters were observed. 
Clinical laboratory data

No clinically relevant shifts from a normal to an abnormal value were observed for haematology, blood chemistry, and urinalysis.

\section{Discussion}

A wide spectrum of drugs is currently available for the treatment of stable angina. Lipid lowering agents, aspirin, nitrates, $\beta$ blockers, and calcium channel blockers all have a role to play, the latter three being relevant for the management of angina symptoms. Under suitable circumstances, nitrates, $\beta$ blockers, and calcium channel blockers can be effective, but there is a good deal of variation in patient response and side effects. Furthermore, nitrates (headaches, flushing, syncope), $\beta$ blockers (unsuitability in asthma, peripheral vascular disease, diabetes), and calcium channel blockers (care in heart failure and poor left ventricular function) have their own characteristic drawbacks. A drug like tedisamil, belonging to none of these classes, offers an alternative treatment.

The findings of our study show tedisamil has anti-ischaemic and antianginal efficacy that is dose dependent and significantly better than placebo. The anti-ischaemic and antianginal effects were more significant at two hours (peak) than 12 hours (trough) after the last dose. The changes from baseline were generally significant for dosages of $100 \mathrm{mg}$ and $150 \mathrm{mg}$ tedisamil twice daily versus placebo, whereas $50 \mathrm{mg}$ tedisamil twice daily differed significantly from placebo in terms of anti-ischaemic effects. The greater efficacy of the two hour over the 12 hour plasma concentration is consistent with the plasma concentration profile of the drug.

These data support a starting dose of tedisamil of $50-100 \mathrm{mg}$ twice daily, since the dose response curve for efficacy parameters shows little or no incremental benefit between $100 \mathrm{mg}$ and $150 \mathrm{mg}$ twice daily. Furthermore, the main side effect of tedisamil, diarrhoea, becomes noticeably more frequent at the $150 \mathrm{mg}$ twice daily dose regimen, which adds support to the 50-100 mg twice daily regimen and provides the optimum safety:efficacy ratio.

Can these findings be explained by the known effects of the drug-bradycardia and/or lengthening of the QT interval? Although tedisamil does show a dose dependent bradycardic effect on coronary artery disease patients at rest, ${ }^{34}$ the effects are smaller during exercise. $^{5}$ In the present study, reductions of 5-15 bpm were seen (unrelated to dose) during exercise, and these findings are consistent with the studies mentioned above. It would seem that some other explanation for the antianginal and anti-ischaemic effects must be proposed. This is unlikely to involve the smooth muscle of coronary vessels, since tedisamil has no consistent effect on blood pressure or peripheral resistance; nor is it likely to involve the myocardium, since no negative inotropic effect has been described. Further preclinical investigations may shed light on other mechanisms.

Since tedisamil makes use of a unique mode of action it is possible that combination treatment with-for example, a long acting nitrate or a calcium channel blocker might provide additive efficacy for the drugs. Controlled clinical trials could test such a hypothesis. In the meantime, our study shows tedisamil to be a safe and effective antianginal and anti-ischaemic drug for the treatment of patients with stable angina.

\section{Appendix}

The Clinical European Studies in Angina and Revascularisation (CESAR 3) investigators were: Dr P Bennett (Bath), Dr R L Blandford (Worksop), Dr T S Callaghan (Brechin), Dr J Chambers (London), Dr K Channer (Sheffield), Dr A J Cowley (Nottingham), Dr C Davidson (Brighton), Dr S Davies (London), Dr J Dhawan (Scunthorpe), Dr I Findlay (Paisley), Dr K Fox (London), Dr M Gammage (Birmingham), Dr B Gould (Sidcup), Dr C Handler (Harrow), Dr J C Kaski (London), Dr A McCance (Derby), Dr MacMahon (Buckinghamshire), Dr M Millar-Craig (Derby), Dr J Morgan (Southampton), Dr A W Nathan (London), Dr D Oakley (Sheffield), Dr W Penney (Cardiff), Dr A Rozkovec (Bournemouth), Dr B Silke (Belfast), Dr Skehan (Leicester), Dr J Stephens (Romford), Dr L Tan (Leeds), Dr A D Timmis (London), Dr C Travell (Luton), and Dr C Ward (Manchester).

1 Dukes ID, Cleeman L, Morad M. Tedisamil blocks the transient and delayed rectifier $\mathrm{K}^{+}$currents in mammalian transient and delayed rectifier $\mathrm{K}^{+}$currents in mammalian cardiac anglial cells. F Pharmacol Exp Ther 1990;254:560-9.
2 Dukes ID, Morad M. The mode of action of tedisamil on voltage dependent $\mathrm{K}^{+}$channels. Cardiovasc Drugs Ther 1992;6:321-7.

3 Mitrovic V, Oehm E, Liebrich A, et al. Haemodynamic and anti-ischaemic effects of tedisamil in humans. Cardiovasc Drugs Ther 1992:6:353-60.

4 Burgheer K, Bode F, Klein V, et al. Prolongation of monophasic action potential duration and the refractory period in the human heart by tedisamil, a new potassium blocking agent. Eur Heart f 1994;15:1409-14.

5 Demolis J-L, Martel C, Funck-Brentano C, et al. Effects of tedisamil, atenolol and their combination on heart and rate-dependent QT interval in healthy volunteers. BrF Clin Pharmacol 1997;44:403-9. 